\title{
Research on Contact State and Its Effect on Forming Precision in Uniform-Contact Stretch Forming Based on Loading at Multi-Position
}

\author{
Lirong Sun ${ }^{1}$, Zhongyi Cai ${ }^{1,2, *}$ and Xiangji $\mathrm{Li}^{1,2, *}$ \\ College of Materials Science and Engineering, Jilin University, Changchun 130025, China \\ 2 Roll Forging Research Institute, Jilin University, Changchun 130025, China \\ * Correspondence: caizy@jlu.edu.cn (Z.C.); xjli@jlu.edu.cn (X.L.); Tel.: +86-0431-8509-4340 (Z.C. \& X.L.)
}

Received: 27 May 2019; Accepted: 24 June 2019; Published: 26 June 2019

\begin{abstract}
Uniform-contact stretch forming based on loading at multi-position (UC-SF) was designed to substitute for conventional stretch forming (C-SF) in the manufacturing of qualified three-dimensional surface parts of a specified shape. Since the integral rigid clamps are replaced by discrete clamps, the sheet metal can be bent in a transverse direction (perpendicular to the stretching direction), and the sheet metal can be automatically warped to the die surface during the loading process. In this paper, finite element numerical simulations were performed to research the contact state evolution and its effect on forming precision by two kinds of loading modes (UC-SF and C-SF). The evolutions of contact state for spherical and saddle-shaped parts were analyzed in different steps, and the results reflect that, in UC-SF, the contact region of curved surface parts is gradually extended in a long strip, and the effective formed regions of the final parts can be in contact with the die surface. However, in C-SF, it is difficult for the final parts to be completely in contact with the die surface, especially spherical parts of a large curvature. Moreover, it is found that the noncontact region of the saddle-shaped part is susceptible to wrinkling in C-SF. Conversely, in UC-SF, the sheet metal can be constrained by contact with a die surface, such that the noncontact region and wrinkle defect disappear and high-precision parts are formed. Finally, stretch forming experiments were carried out and the perfect curved surface part was formed; thus, the experimental results verify the feasibility and effectiveness of UC-SF.
\end{abstract}

Keywords: uniform contact; forming precision; stretch forming; discrete clamps; sheet metal

\section{Introduction}

The stretch forming process, as a significant method to form three-dimensional curved surface parts, has been applied in various industries; for example, the aerospace industry, high-speed train manufacturing, and modern architecture. [1-3]. The sheet metal is clamped by a pair of integral rigid clamps in C-SF; thus, along a transverse direction (perpendicular to stretching direction), it cannot automatically be bent with the curved surface of the die, as shown in Figure 1a. Integral rigid clamps are very stiff and cannot be bent transversely, and can only be loaded in a single position, so the sheet metal is passively in contact with the die surface. Although a great deal of research on optimization of the loading trajectories has been launched, three-dimensional surface parts are prone to produce defects such as noncontact region, wrinkle, crack and severe local deformation in C-SF, especially ones with large curvature [4-7]. In short, how to form superior parts with large curvature and large deformation in the stretch forming process is an interesting topic.

For the purpose of basic process understanding and control, there has been considerable research on C-SF. Parris [8] established a two-dimensional stretch-forming model to predict stress and strain 
distributions, and aimed to improve the precision of the curved parts. Hardt [9] et al. examined various control modes for the stretch-forming process, and found it to be less sensitive to material property. Liu [10] et al. applied the displacement loading method to optimize post-stretching elongation, and reduce forming defects in stretch bending. A precision design method of loading trajectory for longitudinal stretch forming was developed and discussed [11], and the sectional profile in optimal deformation state was extended to define the spatial locations of the discrete clamps. He [12] et al. proposed the transverse stretch forming process to reduce springback of the titanium-alloy skin part, and this model was verified by finite element simulation and experiment. Park [13] used the flexible stretch-forming process based on multiple grippers to form the doubly curved surface parts, and the experiment and numerical simulation results showed that the stretch-forming process can prevent wrinkles from appearing on the curved surface.

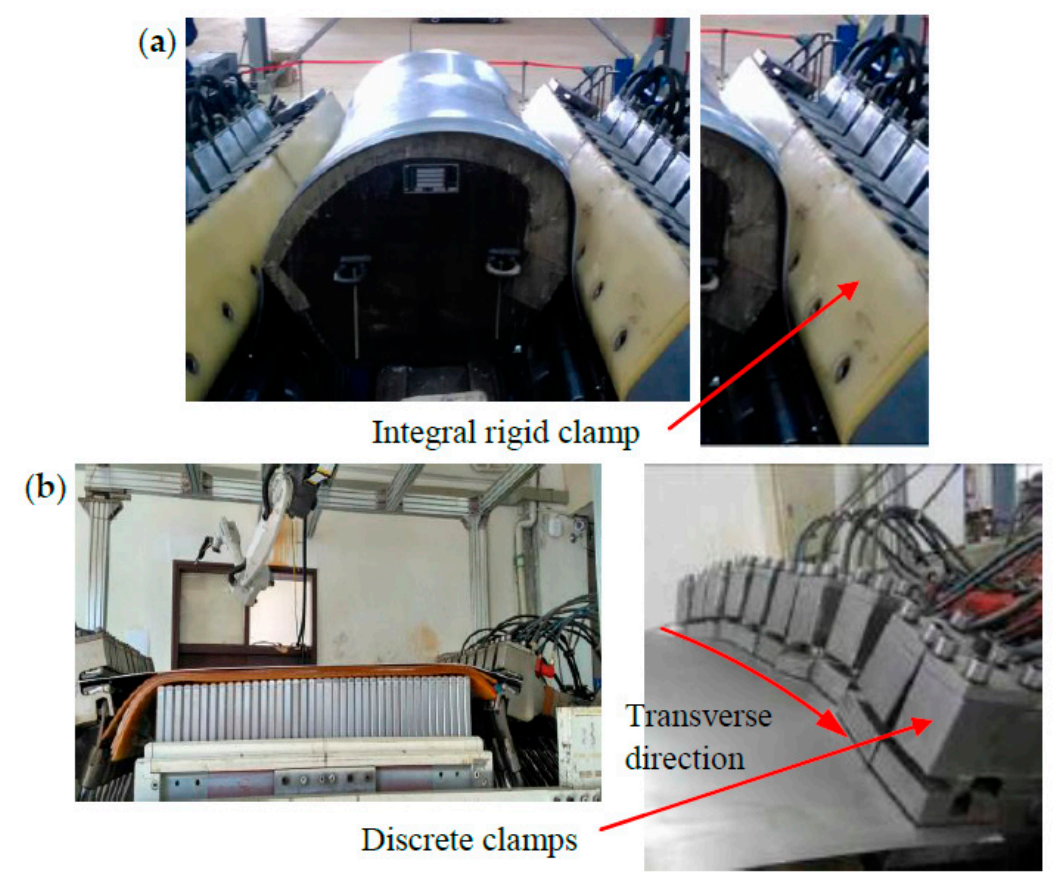

Figure 1. Clamp in stretch forming process. (a) Integral rigid clamp; (b) discrete clamps.

Uniform-contact stretch forming based on loading at multi-position (UC-SF), applying a series of discrete clamps grasped at both ends of the sheet metal, as shown in Figure 1b, is a new method to form three-dimensional curved surface parts. The displacement and rotation angle of each clamp can be individually controlled by discrete loading units, so the sheet metal can be automatically warped to the die surface by loading diverse displacements and rotation angles at multi-positions [14,15]. Cai et al. proposed a new stretch forming process based on minimum deformation path; the method achieved the manufacture of specified shape parts and faultless products of large curvature [16]. In order to explore the optimal moving locus of discrete clamps, a great deal of numerical simulations and experiments were carried out by Yang et al. [17], and the results demonstrate that the strains and stresses of deformed parts presented different distributions when different loading trajectories were adopted. In the stretch forming process, the greater proportion the contact region of effective formed region accounts for, the higher the forming precision is. The contact state between the sheet metal and die has a fundamental effect on quality and forming precision of formed parts; thus, contact state evolution is worthy of further studies and tests [18-21].

The novel contribution of this paper is the design of UC-SF, utilizing FE numerical simulations that examine the contact state evolution between the sheet metal and die surface, and discussing the influence of contact state evolution on forming precision of curved surface parts. In particular, the contact state evolution and its effect on forming precision in different steps are investigated and 
analyzed in C-SF and UC-SF, respectively. The results show that the center and edge region of sheet metal can simultaneously be in contact with the die surface in UC-SF. Moreover, it is proven that UC-SF can ensure that all regions of the sheet metal are in contact with the die surface. The noncontact region and wrinkle defect can be avoided, and high-precision curved surface parts can be formed in UC-SF. The researches indicate that UC-SF can improve the forming precision of curved surface parts by improving the contact state between the sheet metal and the die. Finally, the formed high-quality parts with large curvature can satisfy the practical demands of modern society.

\section{Uniform-Contact Stretch Forming Based on Loading at Multi-Position}

The contact boundary contours between the sheet metal and die are different in the forming process when applying different loading trajectories. The contact regions of the sheet metal can be constrained by die, thus they deform steadily there. Only when all positions of the effective formed region are in contact with the die surface can the contact state between the sheet metal and the die be improved.

Uniform-contact stretch forming based on loading at multi-position was proposed to make the deformation process of the sheet metal more stable. Uniform contact is when the die profile is projected as a rectangle, the contact boundary projection of the sheet metal is consistent with the curve projection of the die, and the contact boundary changes uniformly along the length of the sheet metal during the loading process, as shown in Figure 2a. The perfect contact state can enable the sheet metal to form high-precision curved surface parts, and no defects such as wrinkles, noncontact region and cracking, are generated.


Figure 2. Schematic illustration of the design of UC-SF. (a) Uniform contact; (b) kinetic locus design.

To stretch the die of any profile, the die profile equation is given as $y=f(x, z)$. At arbitrary loading time $t=t_{j}$, the contact boundary curve equation is:

$$
y_{j}^{c}=f\left(x_{j}^{c}, z_{j}^{c}\right)
$$

where, the contact point coordinates $x_{j}^{c}=l_{e} t_{j} / T$, the length of effective formed region is $l_{e}$, and the total loading time is $T$. 
As shown in Figure $2 b$, the arc length of the contact region corresponding to the $i$ th clamp is:

$$
l_{i j}^{c}=\int_{0}^{x_{i j}^{c}} \sqrt{1+[d f(x, z) / d x]^{2}} d x
$$

And the elongation of each longitudinal fiber corresponding to the $i$ th clamp can be found as:

$$
\delta_{i j}=\left(l_{i j}^{c}-x_{i j}^{c}\right) / x_{t_{j}}^{c}
$$

In order to make the strain distribution of the sheet metal more uniform, the total length of the longitudinal fiber corresponding to the ith clamp at $t=t_{j}$ is:

$$
l_{i j}=l_{0}\left(1+\delta_{i j}\right)
$$

where, the initial length of the sheet metal is $l_{0}$, and $l_{0}=l_{e}+l_{t}$, in which $l_{t}$ is the length of transition region, as shown in Figure 2b.

During the loading process, the deformed sheet is always tangent to the die surface in the longitudinal direction, so the rotation angle around $z$-axis of the $i$ th discrete clamp can be calculated by:

$$
\theta_{i j}=\arctan \left[d f\left(x_{i j}, z\right) / d x_{i j}\right]
$$

Based on Equations (1) to (5), the displacements and rotation angle of the $i$ th discrete clamp at a certain loading time $\left(t=t_{j}\right)$ are:

$$
\left\{\begin{array}{l}
\Delta x_{i j}=x_{i j}^{c}+\left(l_{i j}-l_{i j}^{c}\right) \cos \theta_{i j}-l_{0} \\
\Delta y_{i j}=y_{i j}^{c}-\left(l_{i j}-l_{i j}^{c}\right) \sin \theta_{i j}
\end{array}\right.
$$

And the displacement and rotation of the ith clamp from $t=t_{j-1}$ to $t=t_{j}$ is:

$$
\left\{\begin{aligned}
\Delta x_{i j}^{t} & =\Delta x_{i j}-\Delta x_{i(j-1)} \\
\Delta y_{i j}^{t} & =\Delta y_{i j}-\Delta y_{i(j-1)}
\end{aligned}\right.
$$

Moreover, the displacements and rotation angle of the integral clamp in C-SF can be calculated as:

$$
\left\{\begin{array}{l}
\Delta x_{j}=x_{j}^{c}+\left[l_{0}\left(1+\frac{t_{j}}{T} \delta_{\max }\right)-l_{\max }\right] \cos \frac{t_{j}}{T} \theta_{\max }-l_{0} \\
\Delta y_{j}=y_{j}^{c}-\left[l_{0}\left(1+\frac{t_{j}}{T} \delta_{\max }\right)-l_{\max }\right] \sin \frac{t_{j}}{T} \theta_{\max }
\end{array}\right.
$$

where $l_{\max }=\max [l], \delta_{\max }=\max [\delta]$ and $\theta_{\max }=\max [\theta(z)]$, based on Equations (2), (3) and (5).

The loading trajectories of ten discrete clamps for the forming of a spherical part in UC-SF are displayed in Figure 3a, and the loading trajectory of the integral rigid clamp in C-SF is drawn in Figure $3 b$.

Figure 3a shows the loading trajectory of ten discrete clamps in a quarter symmetric model. It can be seen that the loading displacements of the discrete clamps at multi-positions are different, thus realizing the transverse bending deformation of the sheet. However, in C-SF, in order to achieve contact with the die surface, maximum displacement loads were applied to the single integral rigid clamp, as shown in Figure $3 b$. 

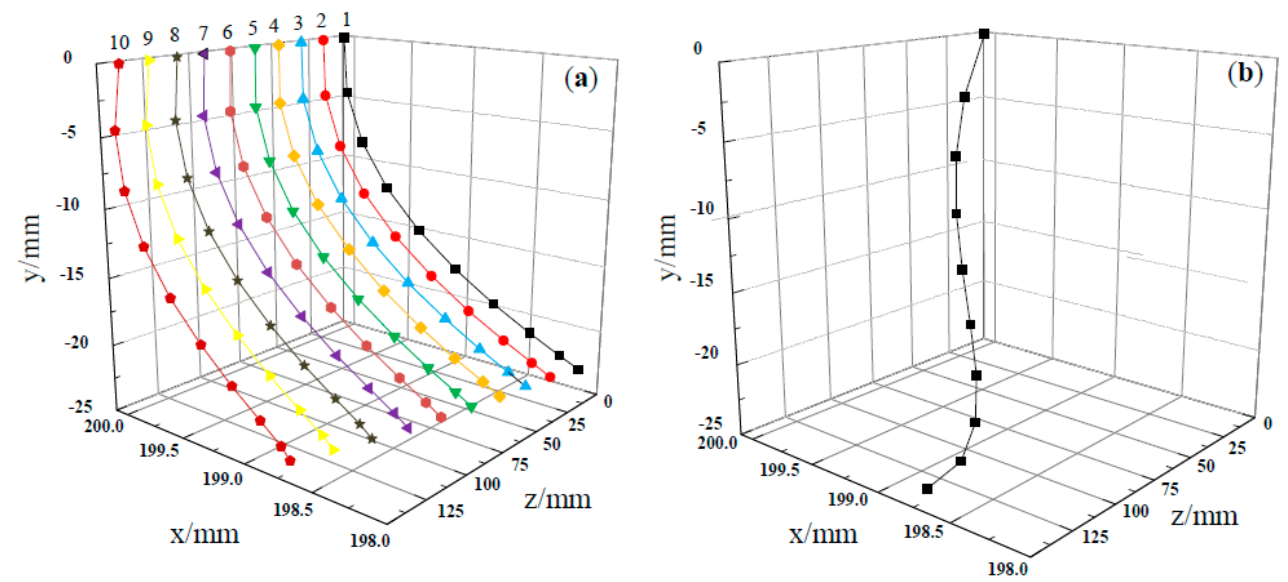

Figure 3. Loading displacements of different clamps in UC-SF and C-SF. (a) UC-SF; (b) C-SF.

\section{Numerical Analysis of Stretch Forming Process}

\subsection{Finite Element Model}

In C-SF, a pair of integral rigid clamps is gripped at both opposite ends of the sheet metal. The straight clamps in C-SF are substituted for a series of discrete clamps in UC-SF. The quarter of FE models were established based on the symmetrical relation of the formed parts, as shown in Figure 4. The numerical simulations were performed with ABAQUS/Explicit (version 6.14, Providence, RI, USA), and five steps were taken in the numerical simulations. As the thickness of the sheet is much smaller than the dimensions of the other directions, the stress in the thickness direction can be ignored, so the sheet metal was modeled with shell elements and meshed with S4R elements. The forming die and the clamps are defined as rigid body and meshed with R3D4 elements; the edges of the clamps are rounded and the mesh is refined to avoid excessive deformation at the grip zone.

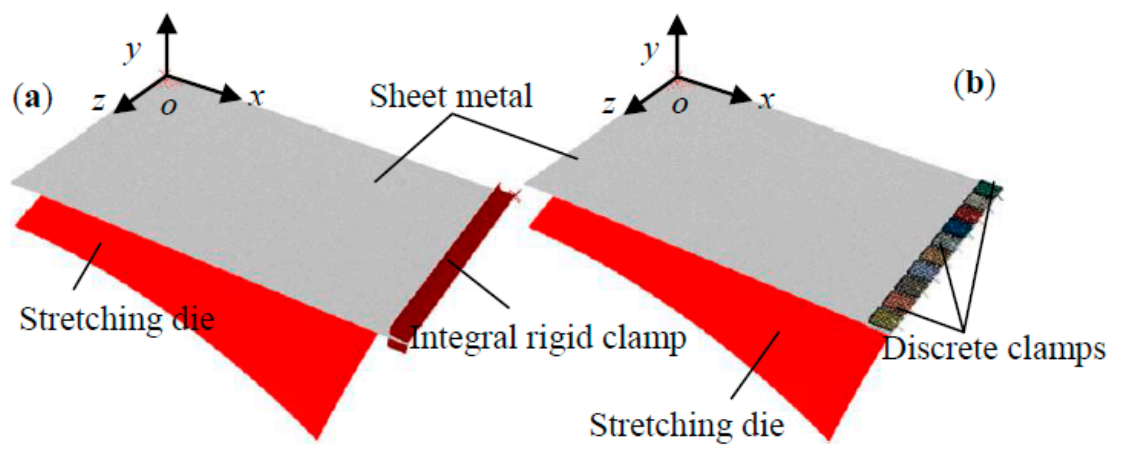

Figure 4. A quarter FE model of stretch-forming. (a) C-SF; (b) UC-SF.

The aluminum alloy sheet of 2024-O was adopted in numerical simulations to study contact state evolution in different analysis steps, and the isotropic, elastic-plastic constitutive behavior with isotropic hardening are assumed for the material. The mechanical properties of the aluminum alloy sheet are listed in Table 1.

Table 1. Mechanical properties of 2024-O aluminum alloy sheet.

\begin{tabular}{cccccc}
\hline $\begin{array}{c}\text { Material } \\
\text { Properties }\end{array}$ & $\begin{array}{c}\text { Density } \\
\left(\mathbf{k g} / \mathbf{m}^{\mathbf{3}} \mathbf{)}\right.\end{array}$ & $\begin{array}{c}\text { Young's Modulus } \\
\mathbf{( G P a )}\end{array}$ & $\begin{array}{c}\text { Poisson's } \\
\text { Ratio }\end{array}$ & $\begin{array}{c}\text { Yield Strength } \\
\mathbf{( M P a})\end{array}$ & $\begin{array}{c}\text { Tensile Strength } \\
\mathbf{( M P a})\end{array}$ \\
\hline Values & 2780 & 40.6 & 0.33 & 75.3 & 191 \\
\hline
\end{tabular}


The contact is a highly nonlinear complex boundary condition. Its simulation should accurately track mutual movement to determine the reasonable and feasible separation conditions for the contact objects. In numerical simulations of the stretch forming process, the contact between the sheet metal and the die surface is defined as general contact. The contact state between the contact pairs is decided by a master-slave algorithm, the surfaces of rigid clamps and rigid die are defined as the master surface, and the surface of the deformed sheet metal is defined as the slave surface.

The contact properties in the normal direction of the sheet metal and clamps, as well as the sheet metal and die are defined as hard contact. In other words, when the normal pressure values between the contact pairs are positive, there is interaction; otherwise, the surfaces of the contact pairs are considered to be in a separated state. In the tangential direction, since there is no relative slip between the sheet metal and clamps during the stretch forming process, rough contact is set, whilst the friction formulation of the sheet metal and die is defined as penalty function. The smaller the friction coefficient is, the smaller the frictional resistance and better fluidity of the sheet metal; which can result in uniform strain and thickness and better quality of the formed part. The penalty parameter is generally $0.1-0.5$; thus, a small friction coefficient between the sheet metal and die was chosen-0.1 [22]. In numerical simulations, once the contact region is penetrated by slave surface in each step of the solution, the penalty function sharply increases the potential energy of the system, such that the system is in an unstable state. Only when the constraint condition is satisfied can the solution of the minimum potential energy principle be solved, thus resolving the contact state.

As shown in Figure 5, if the node of slave surface penetrates the master surface, the normal vector in contact node is calculated as $n_{i}$ and the vector of penetration is $g$; thus, the contact force acting on the contact point can be expressed by:

$$
f_{s}=k_{i} g n_{i}
$$

where $k_{i}$ is the penalty stiffness and can be calculated by

$$
k_{i}=T_{i} K_{i} A_{i}^{2} / V_{i}
$$

where $K_{i}$ is bulk modulus, $V_{i}$ and $A_{i}$ are, respectively, the contact volume and contact area, and $T_{i}$ is the penalty parameter, generally $0.1-0.5$.

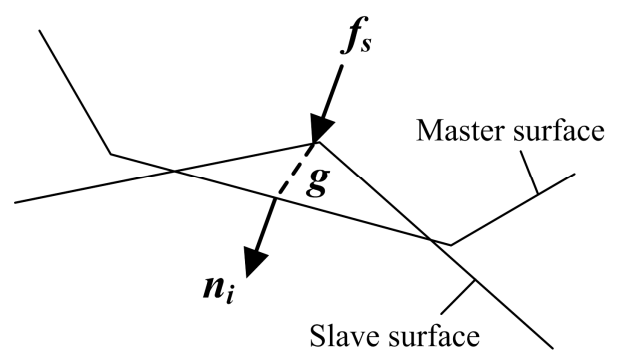

Figure 5. Node penetrates the master surface.

\subsection{Evolution of Contact State between Sheet Metal and Die}

Numerical simulations were carried out for spherical and saddle-shaped parts with Radius $=800 \mathrm{~mm}$ to study the contact state evolution between the sheet metal and the die, and the contact states of the formed parts in different steps were analyzed in C-SF and UC-SF.

In C-SF, it is extremely difficult for the sheet metal with short length of transition region $\left(l_{t}=50 \mathrm{~mm}\right)$ to come into contact with spherical die of Radius $=800 \mathrm{~mm}$ in the final stage. To improve the final contact state, we increase the length of the transition region. When the length of transition region is $100 \mathrm{~mm}\left(l_{t}=100 \mathrm{~mm}\right)$, the effective formed region of the sheet metal can come into contact with the die surface. Figure 6a shows the contact state evolution of spherical part using C-SF in various steps. It was found that the sheet metal is first in contact with the peak of spherical die, while the edge of the 
sheet metal is not in contact with the die surface. With the displacement is loaded, the contact region gradually extends in an elliptical shape from the central region to both edges, and the edge of the sheet metal is in a noncontact separation state for a long time during the loading process. In addition, because the longer transition region is invalid in practical engineering applications, the utilization ratio of the original sheet material is lower, and the economic benefit is greatly reduced.

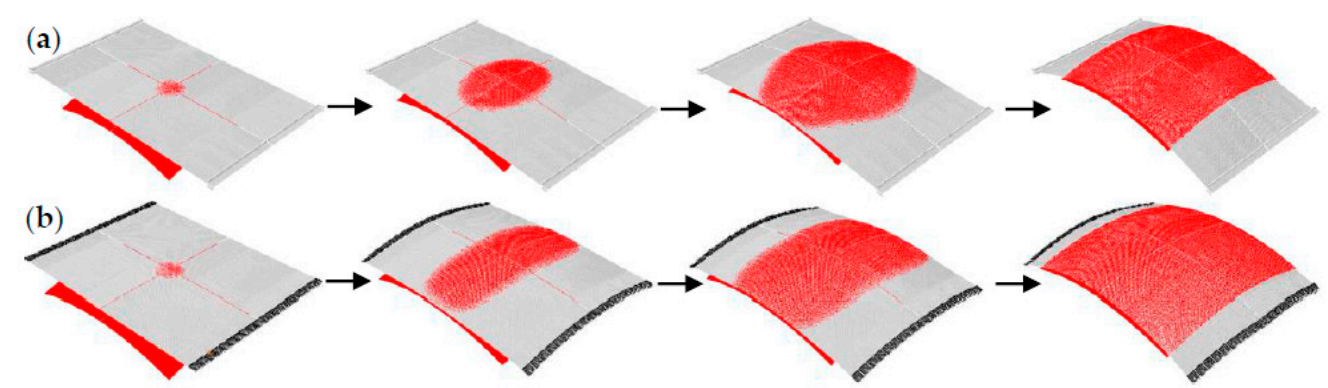

Figure 6. Contact region evolution for spherical parts. (a) C-SF with sheet metal of $l_{t}=100 \mathrm{~mm}$;

(b) UC-SF with sheet metal of $l_{t}=50 \mathrm{~mm}$.

To improve the contact state, UC-SF was designed to achieve rapid contact of the sheet metal with the die surface. Figure $6 \mathrm{~b}$ shows the contact state evolution of different steps in UC-SF to the stretch spherical part of Radius $=800 \mathrm{~mm}$, applying sheet metal with a shorter transition region $\left(l_{t}=50 \mathrm{~mm}\right)$. It can be seen that, at first, the sheet metal can be bent in the transverse direction and maintains a tangent condition with the die surface in the longitudinal direction. As a result, the central and marginal regions of the sheet metal can simultaneously be in contact with the die surface. Then, the contact region is gradually extended into a long rectangular shape until the effective formed region of the sheet metal is in contact with the die surface. Finally, high-precision spherical parts can be obtained after numerical simulations. When reasonable loading trajectories are designed, the sheet metal in the transverse direction can concurrently contact the die surface during the stretch forming process, resulting in avoidance of the noncontact regions, such that more uniform deformation and better formed results are acquired in UC-SF.

On applying the $50 \mathrm{~mm}$-length transition region $\left(l_{t}=50 \mathrm{~mm}\right)$ in C-SF to form saddle-shaped parts of Radius $=800 \mathrm{~mm}$, it can be observed that the center region of the sheet metal cannot come into contact with the saddle-shaped die. When the length of the transition region is increased $\left(l_{t}=100 \mathrm{~mm}\right)$, as expressed in Figure 7a, the sheet metal is first in contact with the edge of the saddle-shaped surface; however, the center region of the sheet metal is in a noncontact separation state during the forming process. Then, the contact region of the saddle-shaped part expands in an arc shape from the edge to the center. Moreover, using a longer initial sheet metal leads to wastage of raw material and reduction of economic benefits. In UC-SF, when the length of transition region is $50 \mathrm{~mm}\left(l_{t}=50 \mathrm{~mm}\right)$, the sheet metal in the transverse direction can simultaneously be in contact with the saddle-shaped die, as shown in Figure $7 \mathrm{~b}$. In addition, the contact region of saddle-shaped part is gradually extended into a long rectangular shape, and all regions of the sheet metal can be in contact with the die surface. The sheet metal can be sufficiently constrained and steadily deformed during the forming process, which makes it easy to obtain perfect three-dimensional surface parts, and applying a shorter transition region length can improve material utilization and economic benefits.

By comparing the contact state evolutions in two kinds of loading modes, it can be observed that when UC-SF is applied to form three-dimensional surface parts, the sheet metal can simultaneously be in contact with the die surface in a transverse direction, and the contact region is gradually extended into long strips. The sheet metal in a noncontact separation state can be avoided in UC-SF, so the final profile of the curved parts can be closer to the target shape and ideal forming results can be obtained. 


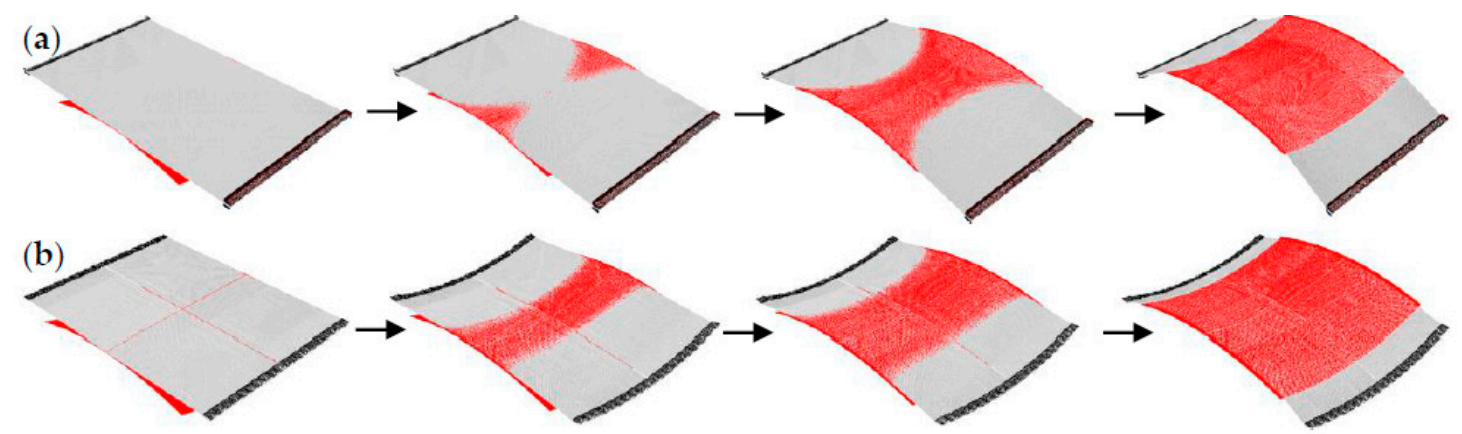

Figure 7. Contact region evolution for saddle-shaped parts. (a) C-SF with sheet metal of $l_{t}=100 \mathrm{~mm}$;

(b) UC-SF with sheet metal of $l_{t}=50 \mathrm{~mm}$.

\section{Effect of Contact State Evolution on Forming Precision in the Stretch Forming Process}

Taking the curved surface parts of large curvatures as an example, numerical simulations were performed in C-SF and UC-SF, and the effect of contact state evolution on forming precision of sheet metal was compared and analyzed.

\subsection{Effect of Contact State Evolution on Forming Precision for Spherical Part}

The evolution of the contact boundary projection on the initial sheet surface with stretch spherical parts of Radius $=400 \mathrm{~mm}$ is shown in Figure 8a. It can be seen that the contact boundary projection changes approximately into an elliptical shape in C-SF, while the edge region of the sheet metal is in a suspended state. And for spherical parts with a large curvature, it is difficult for the four corners of the sheet metal to be in contact with the die surface in the final stage. The existence of noncontact regions may make the forming accuracy of surface parts low and far from the target shape. However, in UC-SF, the contact boundary projection evolves in an approximate rectangular shape, and the sheet metal can be in constant contact with the die. The final spherical part is completely attached to the die, and forming precision is high without any defects.

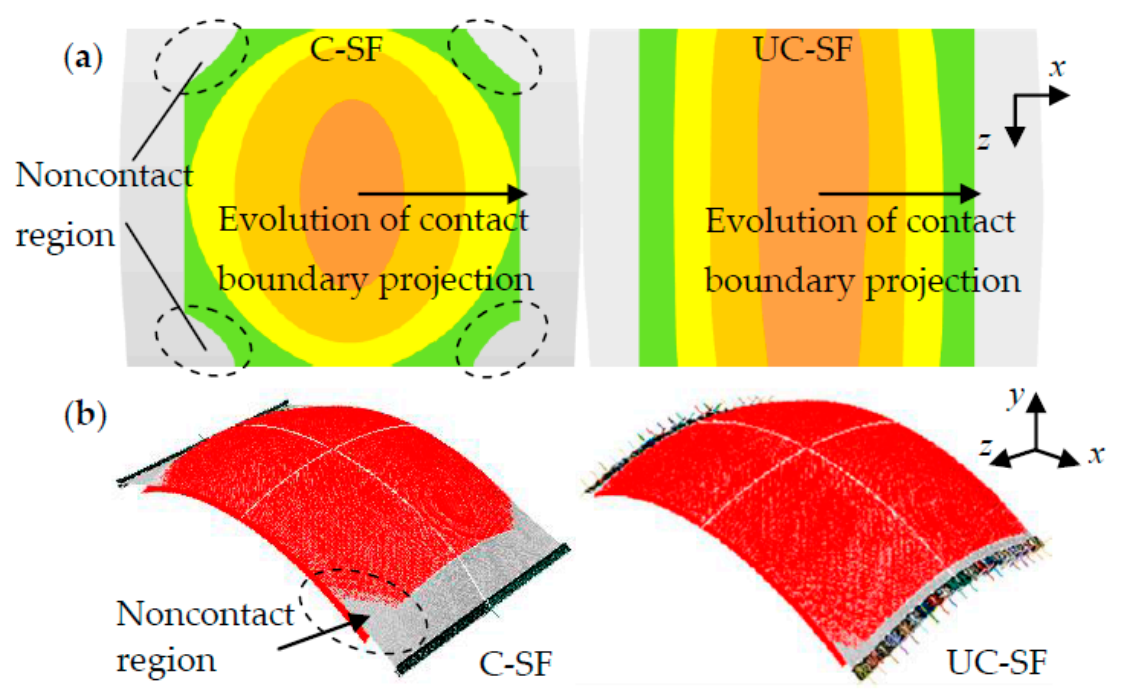

Figure 8. Contact state for spherical part. (a) Contact state evolution; (b) final contact state.

In the loading process, the center region of the sheet metal gradually comes into contact with the die, but the quadrilateral edge region of the sheet metal is in a noncontact separation state in C-SF, as shown in Figure 8b. Especially for curved parts with large curvature, the sheet metal cannot be in contact with the die surface, a phenomenon that is easy to produce. However, in UC-SF, because the different displacements and rotation angles loaded at discrete clamps can enable the transverse bending 
of the sheet metal, the edge and center region of the sheet metal can be simultaneously in contact with the die surface. A better curved surface can be obtained by using a shorter length of transition region in UC-SF; in other words, qualified surface parts can be acquired, while saving on material.

In order to more intuitively analyze the loading process, the coordinate data in longitudinal edge lines of the spherical part and the die are respectively extracted in different analysis steps, and the curves drawn are as shown in Figure 9. As can be seen from Figure 9a, when stretching the spherical part of large curvature in C-SF, the edge of the sheet metal is not attached to the die surface, and the longitudinal fiber of the sheet metal does not maintain a tangent relationship with the die surface. Conversely, in UC-SF, the longitudinal fiber of the sheet metal is always tangent to the die surface until the sheet metal and the die are completely in a contact state, as shown in Figure $9 \mathrm{~b}$. In conclusion, the UC-SF can enable a contact state between the sheet metal and the die, and high-precision spherical parts can thus be formed.
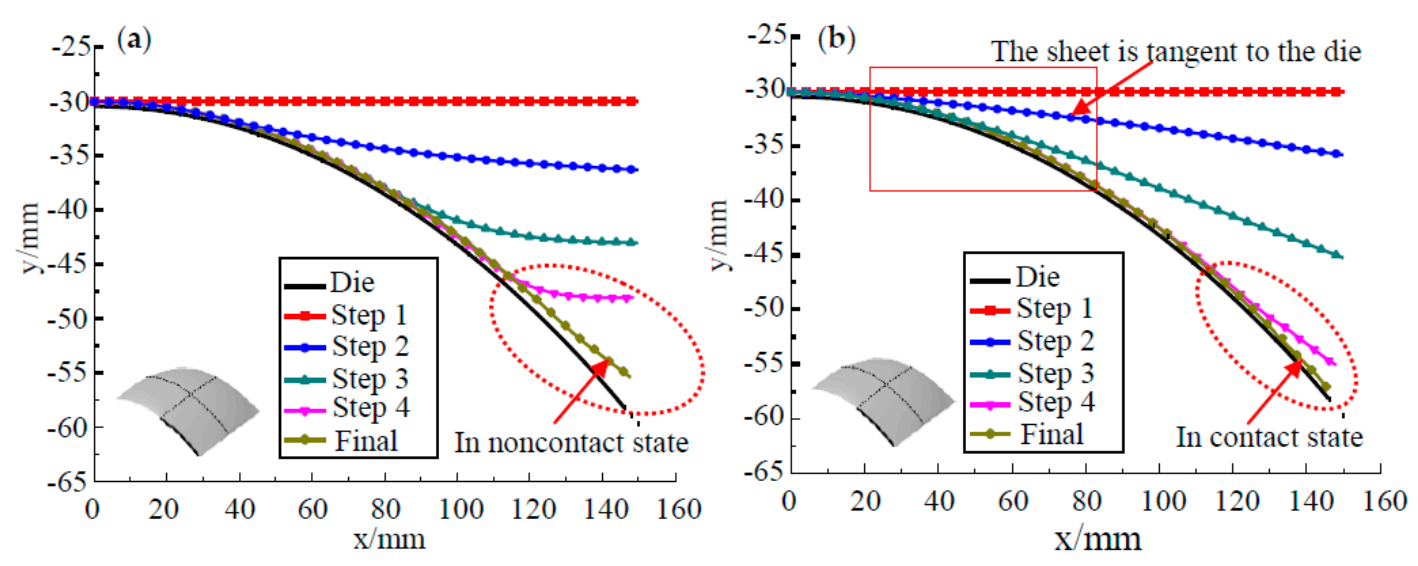

Figure 9. Longitudinal section lines of spherical part in the effective formed region. (a) C-SF; (b) UC-SF.

Figure 10 shows the gap between the edge of the final spherical parts and the die. It can be seen that the sheet and the die are completely fitted in UC-SF. However, in C-SF, there is a certain gap in the noncontact region between the sheet metal and the die-about $2-7 \mathrm{~mm}$. The closer to the edge portion of the spherical part, the larger the gap between the sheet and the die is. The presence of the gap reduces the accuracy of the forming part, and the precision of the formed part is lower in the larger gap.

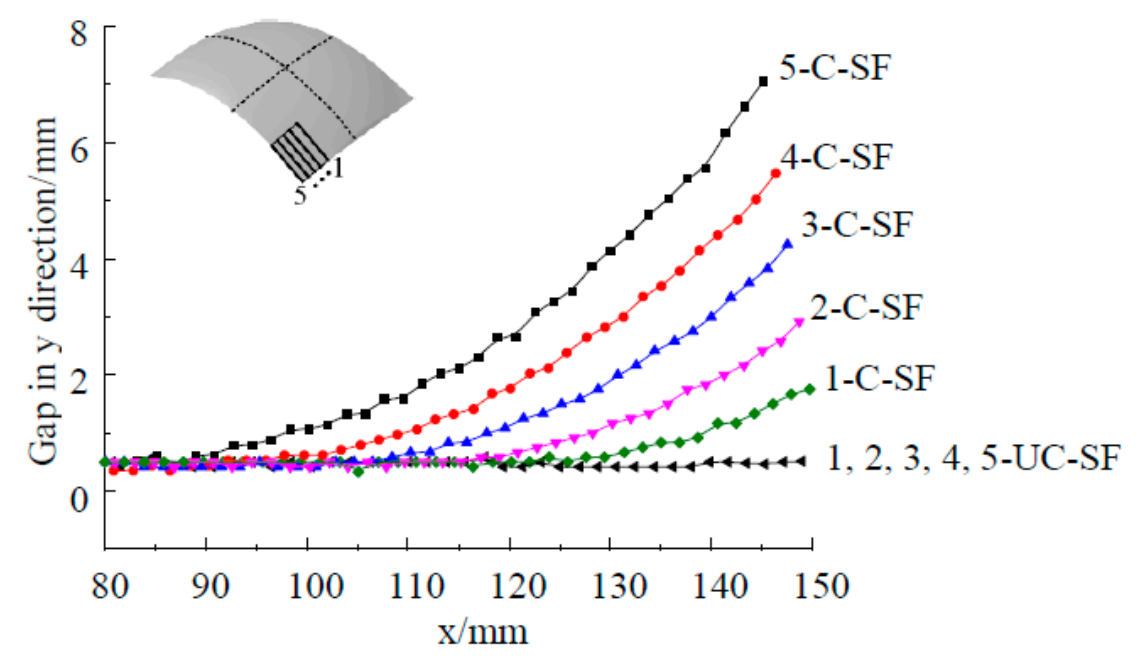

Figure 10. Gap between final sheet metal and die in different positions. 


\subsection{Effect of Contact State Evolution on Forming Precision for the Saddle-Shaped Part}

\subsubsection{Wrinkle Evolution for the Saddle-Shaped Part}

Figure 11a shows the evolution of the contact boundary projection on the initial sheet surface, when the saddle-shaped part of the large curvature is formed. It is difficult for the center region of the saddle-shaped part with Radius $=400 \mathrm{~mm}$ to come into contact with the die, and wrinkle defects appear in the noncontact region. As the contact region gradually expands, it is gradually constrained by the die, and the wrinkles gradually decrease in the noncontact region. However, in UC-SF, since the sheet metal loaded by discrete clamps can be bent into an arbitrary shape in the transverse direction, the center position of the saddle-shaped part is in contact with the die at the beginning of the deformation, and the contact region expands into a rectangular shape. Finally, since the sheet metal is completely restrained by the die, the wrinkle defect disappears, and a high-precision saddle-shaped part can be formed.

(a)

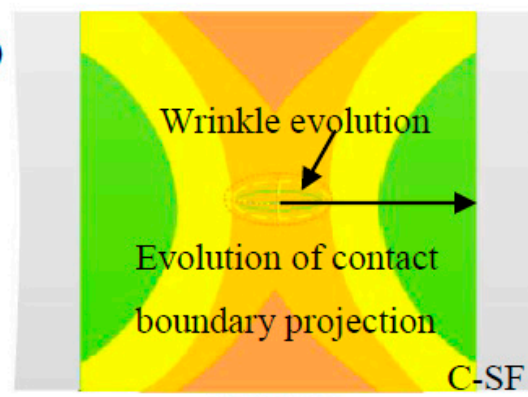

(b)

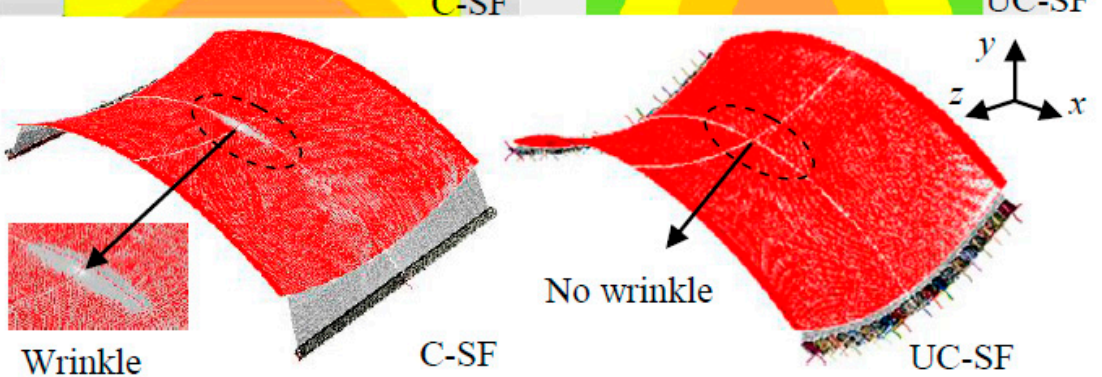

Figure 11. Contact state for saddle-shaped part. (a) Contact state evolution; (b) final contact state.

When stretching the saddle-shaped part of Radius $=400 \mathrm{~mm}$ in C-SF, wrinkles appear in the center region, as shown in Figure 11b. Partial elements in the noncontact separation state were compressed in the transverse direction and out-plane buckling, leading to the occurrence of wrinkling in the center. On the contrary, no wrinkling occurs in the saddle-shaped part, when UC-SF is adopted to form saddle-shaped parts of large curvature. The sheet metal is in contact with the die during the deformed process, which can ensure that the saddle-shaped part is sufficiently constrained by the die, effectively restraining wrinkle defects, and meeting the quality requirements of actual production.

The position coordinates in transverse center lines of the saddle-shaped part and the die profile are extracted, as show in Figure 12, and the occurrence of wrinkling is analyzed by observing the deviation of profile line contours. In Figure 12a, in C-SF, the central region of the sheet metal cannot be in contact with the die, and wrinkles are produced because of unstable plastic deformation. Under different analysis steps, the wrinkle height of the sheet metal gradually decreases, but the final formed part still shows obvious wrinkles. However, in UC-SF, the sheet metal can be bent laterally, so the sheet metal can come into contact with the central region of the saddle-shaped die first, and the sheet metal is constrained by the die. Moreover, in the subsequent loading process, the transverse center line of the sheet metal is always in contact with the die, as shown in Figure 12b, and the sheet metal is stably deformed. Finally, the qualified saddle-shaped part of large curvature is obtained. 

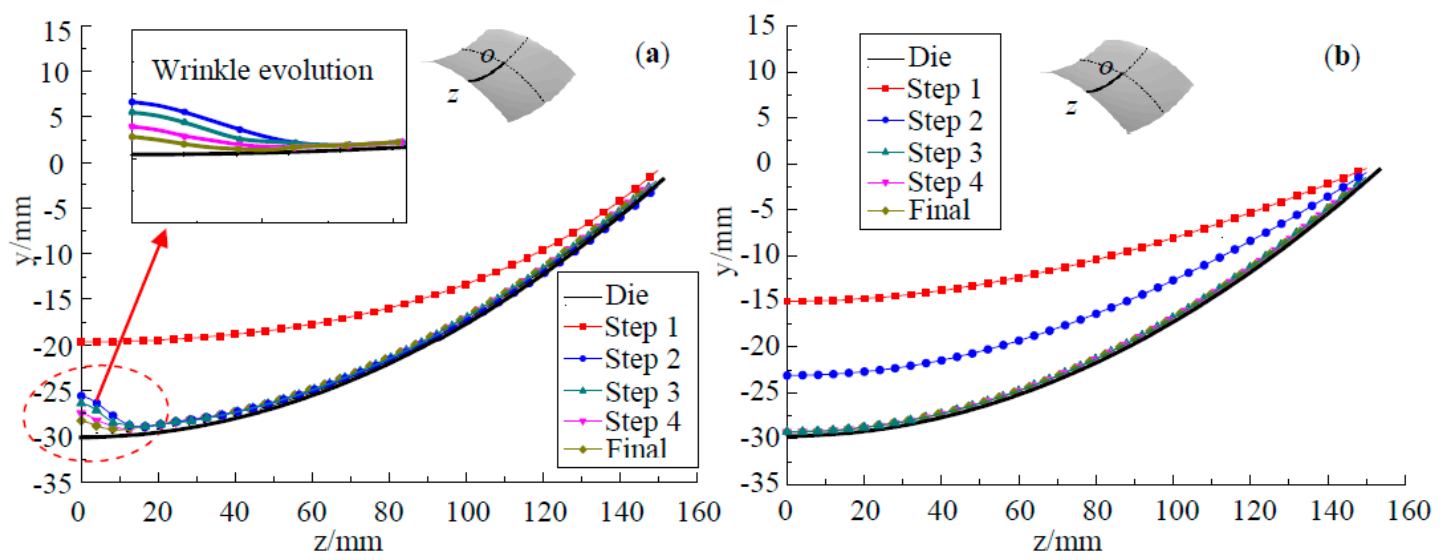

Figure 12. Transverse section lines of the saddle-shaped part in different steps. (a) C-SF; (b) UC-SF.

4.2.2. Wrinkle Analysis for the Saddle-Shaped Part

In C-SF, transverse stress distributions are uneven in the saddle-shaped part, and larger transverse compressive stresses appear in the center region. Wrinkles occur in the critical region when the transverse compressive stress exceeds a certain critical value, as shown in Figure 13a. However, in UC-SF, the transverse compressive stresses are always less than the critical value, and the variations of transverse stresses are relatively gentle; thus, wrinkles do not occur in saddle-shaped parts when the transverse compressive stress of the sheet metal is very small (Figure 13b).
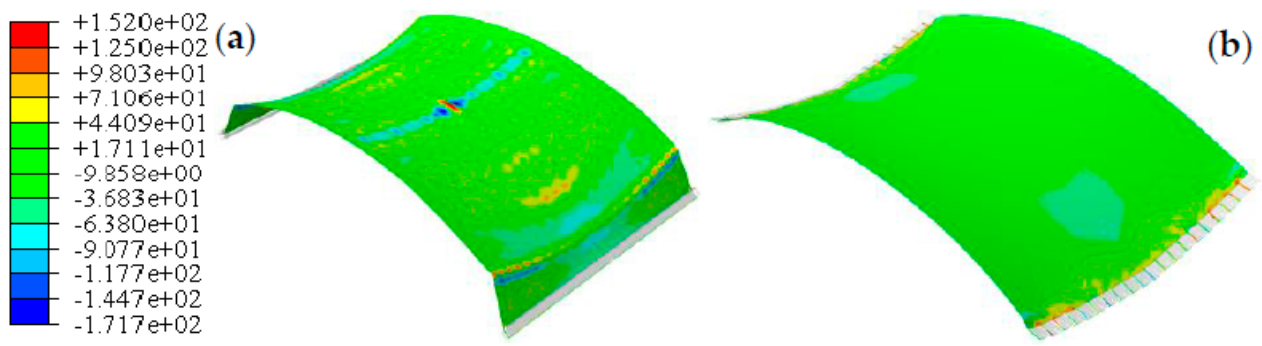

Figure 13. Transverse stresses distributions of the saddle-shaped part. (a) C-SF; (b) UC-SF.

By extracting the transverse stress at the center line of the saddle-shaped part, as shown in Figure 14a, it can be seen that, in C-SF, the transverse stress changes drastically between the tensile and compressive stress at different loading steps, and there is large compressive stress more than the critical value on both sides of the wrinkle area. Larger transverse compressive stresses appear in the critical region that is subjected to wrinkles in C-SF, which is the main cause of wrinkling instability. As the loading progresses, the transverse compressive stress regions gradually shift toward the center region of the sheet metal, and the width of the wrinkles gradually decreases. There is large tensile stress in the wrinkle area, which may cause the saddle-shaped part to crack during the forming process. However, in UC-SF, the transverse compressive stress slightly changes, as shown in Figure 14b, and the sheet metal is able to maintain the small compressive stress. Smaller compressive stresses appear in the critical region, and the sheet metal is completely constrained by the die, so no wrinkle defects appear.

In conclusion, it is difficult for the sheet metal to completely come into contact with the die surface in C-SF, and the large-curvature parts may produce some defects, such as noncontact regions and wrinkles. The shape error of the formed part is large in the noncontact region, the forming precision is low, and the actual requirements are not satisfied in C-SF. However, when the loading trajectory is reasonably designed, all regions of the sheet metal can simultaneously come into contact with the die surface, the deformed parts get sufficient constraint from the die, and the forming precision of curved parts can be improved in UC-SF. 

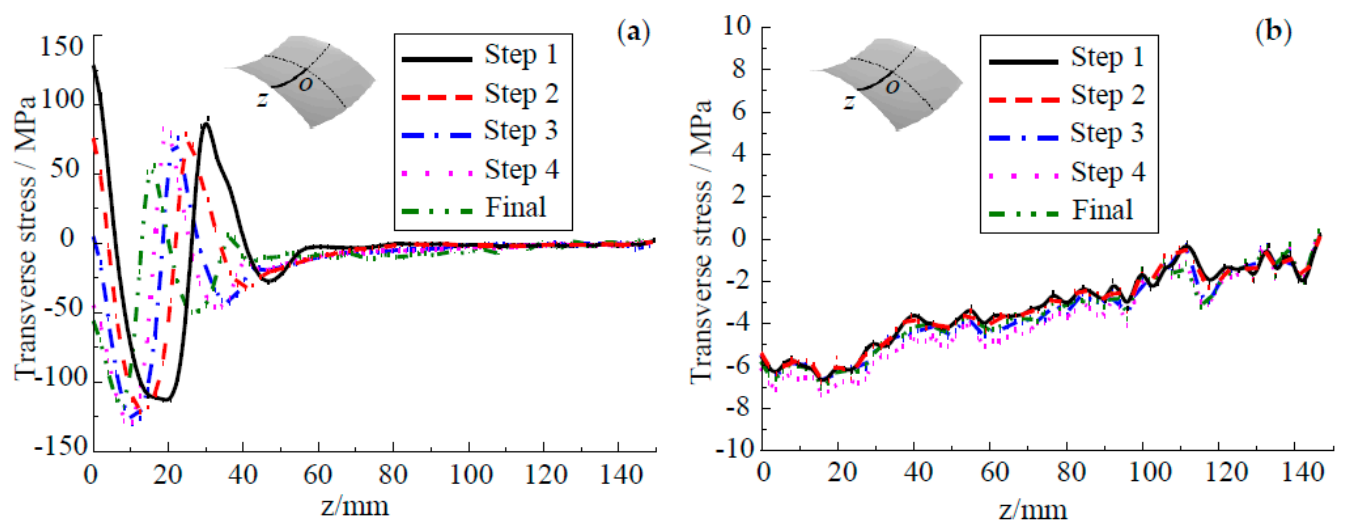

Figure 14. Transverse stresses of the saddle-shaped part at different steps. (a) C-SF; (b) UC-SF.

\section{Experimental Validation}

In order to verify the feasibility of the designed loading locus in UC-SF, the experiments for the forming of spherical parts were carried out on a home-made apparatus at Jilin University. As shown in Figure $1 \mathrm{~b}$, the discrete clamps can be assembled by an array of adjacent jaws, and can be curved from a straight line up to an arbitrary curvature. An aluminum 2024-O sheet metal is applied to form the target with a radius of curvature $800 \mathrm{~mm}$. The length and width of the sheet metal in the experiments are $1500 \mathrm{~mm}, 1000 \mathrm{~mm}$ and $1 \mathrm{~mm}$ in thickness, respectively; the length and width of the effective formed region are $1000 \mathrm{~mm}$ and $1000 \mathrm{~mm}$.

The spherical part in experiment is shown in Figure 15a. It can be observed that the effective formed region of the spherical part is of good surface quality and without any defects. The sheet metal can be in contact with the die surface, and the stretch forming process can be carried out quickly and smoothly. The experiment results demonstrate that the qualified three-dimensional surface parts can be acquired and used in engineering manufacture when UC-SF is adopted. The shape errors between the experimental results and the objective shape are shown in Figure 15b. It can be observed that the shape error of spherical parts in UC-SF mainly ranged from $-1 \mathrm{~mm}$ to $1 \mathrm{~mm}$, and the absolute values of errors on more than $90 \%$ of the area of the formed part are less than $1 \mathrm{~mm}$.
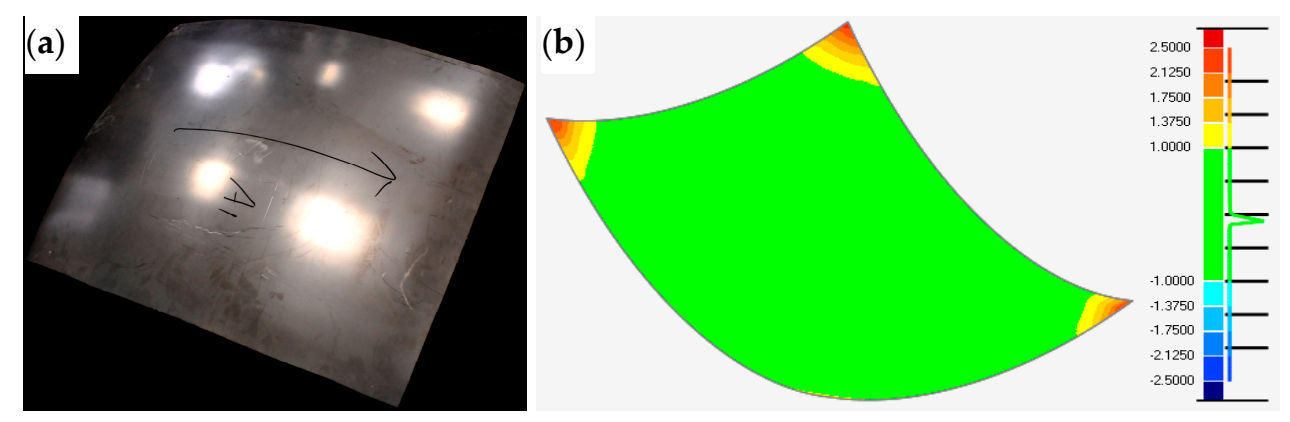

Figure 15. Spherical part in the experiment. (a) Spherical part; (b) shape error distribution in a normal direction.

The real-time locus of each discrete clamp can be calculated by Equation (6), and contact state of the deformed sheet and die at a specific intermediate time for the experiment was shown in Figure 16. The desirable state of the stretched sheet metal is in accordance with the configuration of the die surface by adjustment of each discrete clamp. In this scenario, the entire sheet metal was in contact with the die surface during the forming process, increasing the profile accuracy of the curved surface parts. 


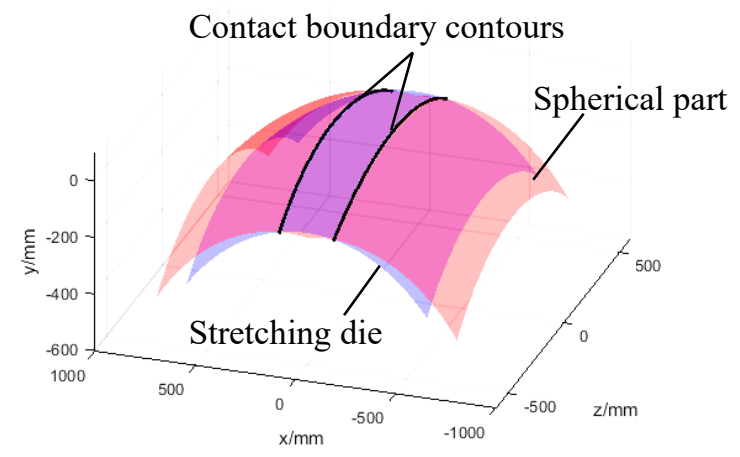

Figure 16. Contact state between the deformed sheet metal and the die.

\section{Conclusions}

To research contact state evolution between the sheet metal and the die surface and its effect on forming precision of sheet metal in stretch forming process, stretching spherical and saddle-shaped parts were numerically analyzed in UC-SF and C-SF. And the results of the relevant study in this paper are summarized as follows:

1. In order for the effective formed region of the sheet metal to simultaneously be in contact with the die surface, uniform-contact stretch forming based on loading at multi-positions was designed. The entire sheet metal can be in contact with the die by loading reasonable displacements and rotation angles at multiple discrete clamps.

2. The evolution of contact region: In C-SF, the spherical and saddle-shaped parts are firstly in contact with the center and edge region of the die, respectively. Then, the contact region of the spherical parts constantly expands in an elliptical shape from the center to the edge; conversely, that of the saddle-shaped parts constantly expands in an arc shape from the edge to the center, whilst the edge of the spherical part and the central region of the saddle-shaped part are in a noncontact separation state during the stretch forming process. However, in UC-SF, the contact regions of the spherical parts and saddle-shaped parts are extended into a long rectangular shape, and all regions of the effective formed regions are simultaneously in contact with the die surface.

3. In UC-SF, the longitudinal fibers of the sheet metal maintain a tangent relationship with the die surface during the stretching process, and the edge and center regions of the sheet metal can be simultaneously in contact with the die surface, such that high-precision curved parts of large curvature can be obtained. However, in C-SF, the quadrilateral edge region of the spherical part is in a noncontact separation state, and there is a certain gap in the noncontact region; it is difficult for the center region of the saddle-shaped part to come into contact with the die, and wrinkles occur in the noncontact region, when the transverse compressive stress is more than the critical value. Noncontact regions and wrinkle defects can reduce the forming accuracy of the curved parts.

4. The experiments carried out on the home-made apparatus have verified the feasibility of the UC-SF. The results indicate that high-quality curved parts with large curvature can be manufactured in UC-SF, when loading locus is properly adopted.

Author Contributions: Conceptualization, L.S. and Z.C.; Methodology, L.S. and Z.C.; Software, L.S.; Validation, L.S., Z.C. and X.L.; Investigation, L.S., Z.C. and X.L.; Data curation, L.S. and X.L.; Funding acquisition, Z.C.; Writing-original draft preparation, L.S.; Writing-review and editing, L.S. and Z.C.

Funding: This research was funded by the National Natural Science Foundation of China (Grant No. 51575231).

Conflicts of Interest: The authors declare no conflict of interest.

\section{References}

1. Wang, S.H.; Cai, Z.Y.; Li, M.Z. Numerical investigation of the influence of punch element in multi-point stretch forming process. Int. J. Adv. Manuf. Technol. 2010, 49, 475-483. [CrossRef] 
2. Xing, J.; Cheng, Y.Y.; Yi, Z. The effect of swinging ball heads with different arrangements in multi-point stretch-forming process. Material 2019, 12, 337. [CrossRef]

3. Kuboki, T.; Jin, Y.; Murata, M. Prediction of stress-strain diagram from forming load in stretch forming. Int. J. Mech. Sci. 2012, 60, 46-53. [CrossRef]

4. Cai, Z.Y.; Wang, S.H.; Li, M.Z. Numerical investigation of multi-point forming process for sheet metal: Wrinkling, dimpling and springback. Int. J. Adv. Manuf. Technol. 2008, 37, 927-936. [CrossRef]

5. Anagnosou, E.L.; Papazian, J.M. Optimized tooling design algorithm for sheet metal forming over reconfigurable compliant tooling. AIP Conf. Proc. 2004, 712, 741-748.

6. Wang, Y.; Li, M.Z. Research on three-dimensional surface parts in multi-gripper flexible stretch forming. Int. J. Adv. Manuf. Technol. 2014, 71, 1701-1707. [CrossRef]

7. Siegert, K.; Rennet, A.; Fann, K.J. Prediction of the final part properties in sheet metal forming by CNC-controlled stretch forming. J. Mater. Proc. Technol. 1997, 71, 141-146. [CrossRef]

8. Parris, A. Precision stretch forming for precision assembly. Ph.D. thesis, Massachusetts Institute of Technology, Cambridge, MA, USA, 1996.

9. Hardt, D.E.; Chung, K.; Richmond, O. In process control of strain in a stretch forming process. J. Eng. Mater. Technol. 2001, 123, 497-503. [CrossRef]

10. Liu, C.G.; Zhang, X.G.; Wu, X.T.; Zheng, Y. Optimization of post-stretching elongation in stretch bending of aluminum hollow profile. Int. J. Adv. Manuf. Technol. 2016, 82, 1937-1946. [CrossRef]

11. Peng, J.W.; Li, W.D.; Han, J.Q.; Wan, M.; Meng, B. Kinetic locus design for longitudinal stretch forming of aircraft skin components. Int. J. Adv. Manuf. Technol. 2016, 86, 3571-3582. [CrossRef]

12. He, D.; Li, D.; Li, X.; Jin, C. Optimization on springback reduction in cold stretch forming of titanium-alloy aircraft skin. Trans. Nonferous Metals Soc. China 2010, 12, 2350-2357. [CrossRef]

13. Park, J.W.; Kim, J.; Kim, K.H.; Kang, B.S. Numerical and experimental study of stretching effect on flexible forming technology. Int. J. Adv. Manuf. Technol. 2014, 73, 1273-1280. [CrossRef]

14. Cai, Z.Y.; Wang, S.H.; Xu, X.D.; Li, M.Z. Numerical simulation for the multi-point stretch forming process of sheet metal. J. Mater. Proc. Technol. 2009, 209, 396-407. [CrossRef]

15. Cai, Z.Y.; Liang, X.B.; Yang, Z.; Li, X.J. Force-controlled sheet metal stretch forming process based on loading at discrete points. Int. J. Adv. Manuf. Technol. 2017, 93, 1781-1789. [CrossRef]

16. Cai, Z.Y.; Yang, Z.; Che, C.J.; Li, M.Z. Minimum deformation path sheet metal stretch forming based on loading at discrete points. Int. J. Adv. Manuf. Technol. 2016, 87, 2683-2692. [CrossRef]

17. Yang, Z.; Cai, Z.Y.; Che, C.J.; Li, M.Z. Numerical simulation research on the loading trajectory in stretch forming process based on distributed displacement loading. Int. J. Adv. Manuf. Technol. 2016, 82, 1353-1362. [CrossRef]

18. Pereira, M.P.; Yan, W.; Rolfe, B.F. Contact pressure evolution and its relation to wear in sheet metal forming. Wear 2008, 265, 1687-1699. [CrossRef]

19. Neale, K.W.; Tugcu, P. Numerical analysis of wrinkle formation tendencies in sheet metals. Int. J. Num. Meth. Eng. 1990, 30, 1595-1608. [CrossRef]

20. Shim, H.; Suh, E. Contact treatment algorithm for the trimmed NURBS surface. J. Mater. Proc. Tech. 2000, 104, 200-206. [CrossRef]

21. Kim, T.J.; Yang, D.Y. FE-analysis of sheet metal forming processes using continuous contact treatment. Int. J. Plastic. 2007, 23, 544-560. [CrossRef]

22. Peng, H.L. Research on process and numerical simulation of flexible clamp stretch forming. Ph.D. thesis, Jilin University, Changchun, China, 2013.

(C) 2019 by the authors. Licensee MDPI, Basel, Switzerland. This article is an open access article distributed under the terms and conditions of the Creative Commons Attribution (CC BY) license (http://creativecommons.org/licenses/by/4.0/). 